\title{
ERRATUM
}

\section{Allogeneic donor bone marrow cells recovery and infusion after allogeneic face transplantation from the same donor}

\author{
O Hequet ${ }^{1}$, E Morelon ${ }^{2}$, J-P Bourgeot ${ }^{1}$, V Dubois ${ }^{3}$, B Devauchelle ${ }^{4}$, J-M Dubernard ${ }^{2}$ and M Michallet ${ }^{5}$ \\ ${ }^{1}$ Laboratory of Cellular Therapy, EFS, Edouard Herriot Hospital, Lyon, France; ${ }^{2}$ Department of Transplantation, Edouard Herriot \\ Hospital, UCLB1, Lyon, France; ${ }^{3}$ HLA Laboratory, EFS, Lyon, France; ${ }^{4}$ Department of Facial Surgery, Amiens Picardie Hospital, \\ Amiens, France and ${ }^{5}$ Department of Hematology, Edouard Herriot Hospital, Lyon, France
}

Bone Marrow Transplantation (2008) 42, 143; doi:10.1038/bmt.2008.202

Correction to: Bone Marrow Transplantation (2008) 41, 1059-1061; doi:10.1038/bmt.2008.23

The order of authors' names was published incorrectly. The correct author list and affiliations are shown above.

We would like to apologize for the error. 